\title{
A CLASSICAL APPROACH TO EIGENVALUE PROBLEMS ASSOCIATED WITH A PAIR OF MIXED REGULAR STURM-LIOUVILLE EQUATIONS I
}

\author{
M. VENKATESULU and PALLAV KUMAR BARUAH \\ Sri Sathya Sai Institute of Higher Learning \\ Department of Mathematics and Computer Science \\ Prasanthinilayam 515134 \\ Andhra Pradesh, India
}

(Received February, 1995; Revised December, 1999)

In the studies of acoustic waveguides in ocean, buckling of columns with variable cross sections in applied elasticity, transverse vibrations in non homogeneous strings, etc., we encounter a new class of problems of the type $L_{1} y_{1} \overline{\overline{2}}-\frac{d^{2} y_{1}}{d x^{2}}+q_{1}(x) y_{1}=\lambda y_{1}$ defined on an interval $\left[d_{1}, d_{2}\right]$ and $L_{2} y_{2}=-\frac{d^{2} y_{2}}{d x^{2}}+q_{2}(x) y_{2}=\lambda y_{2}$ on the adjacent interval $\left[d_{2}, d_{3}\right]$ satisfying certain matching conditions at the interface point $x=d_{2}$.

Here in Part I, we constructed a fundamental system for $\left(L_{1}, L_{2}\right)$ and derive certain estimates for the same. Later, in Part II, we shall consider four types of boundary value problems associated with $\left(L_{1}, L_{2}\right)$ and study the corresponding spectra.

Key words: Sturm-Liouville Equations, Interface Boundary Conditions, Initial Value Problems, Matching Conditions, Fundamental System, Eigenvalue Problems, Estimates, Inequalities.

AMS subject classifications: $34 \mathrm{XX}, 34 \mathrm{~A} 10,34 \mathrm{~A} 15,34 \mathrm{~A} 30$.

\section{Introduction}

In studies of acoustic waveguides in ocean [1], buckling of columns with variable cross sections in applied elasticity [9], transverse vibrations in nonhomogeneous strings [2], etc., we encounter a new class of problems of the type

$$
L_{1} y_{1}=-\frac{d^{2} y_{1}}{d x^{2}}+q_{1}(x) y_{1}=\lambda y_{1}
$$

defined on an interval $\left[d_{1}, d_{2}\right]$ and

$$
L_{2} y_{2}=-\frac{d^{2} y_{2}}{d x^{2}}+q_{2}(x) y_{2}=\lambda y_{2}
$$


defined on the adjacent interval $\left[d_{2}, d_{3}\right]$, where $\lambda$ is an unknown constant (eigenvalue) and the functions $y_{1}, y_{2}$ are required to satisfy certain matching conditions at the interface $x=d_{2}$. In most of the cases, the complete set of physical conditions give rise to (selfadjoint) eigenvalue problems associated with the pair $\left(L_{1}, L_{2}\right)$. The spectral analysis of these boundary value problems (BVPs) can be carried out to some extent by recasting them as operator equations in an appropriate abstract space [3, 8]. But some of the nice and useful properties of the original BVPs cannot be captured so easily in the abstract space settings. In the literature, there do not seem to exist may results in this area. However, O.H. Hald [5] discusses the inverse theory of some problems of this type which arise in torsional modes of the Earth, and B.J. Harris [6] obtains series solutions for certain Riccatti equations with applications to Sturm-Liouville problems

Hence here and in the sequel, we adopt the classical approach for the study of eigenvalue problems (EVPs) associated with the pair $\left(L_{1}, L_{2}\right)$ and prove a few spectral analysis results for the new class of BVPs.

Before proceeding to the work, we shall introduce a few notations and definitions. Let $R$ denote the real line, and $C$ denote the complex plane with their usual topologies. For a complex number $\lambda, \operatorname{Re} \lambda$ and $\operatorname{Im} \lambda$ denote the real and imaginary parts of $\lambda$, respectively. For any two nonempty sets $A$ and $B, A \backslash B$ denotes the collection of elements in $A$ which are not in $B$. Again, for any two nonempty sets $V_{1}$ and $V_{2}, V_{1} \times V_{2}$ denotes the Cartesian product (space equipped with the product topology) of $V_{1}$ and $V_{2}$, taken in that order. For a compact interval $[a, b]$, of $R$, $L_{C}^{2}[a, b]\left(L_{R}^{2}[a, b]\right)$ denotes the complex (real) Hilbert space of all complex (real) valued Lebesgue square integrable functions defined on $[a, b]$. The inner product $(\cdot, \cdot)$ and norm $\|\cdot\|$ in $L_{C}^{2}[a, b]\left(L_{R}^{2}[a, b]\right)$ are given by

$$
(f, g)=\int_{a}^{b} f \bar{g} d x \text { and }\|f\|=(f, f)^{1 / 2}
$$

where $\bar{g}$ denotes the complex conjugate of $g$. For a function $y, y^{\prime}$ and $y^{\prime \prime}$ denote the first and second order derivatives of $y$, respectively, if they exist. Let $A C^{2}[a, b]$ denote the space of all twice continuously differentiable complex valued functions $y$ defined on $[a, b]$ such that $y^{\prime}$ is absolutely continuous. Let $H_{C}^{2}[a, b]$ denote those functions $y \in A C^{2}[a, b]$ such that $y^{\prime \prime} \in L_{C}^{2}[a, b]$. Let $0<h<1$ and let $\left(q_{1}, q_{2}\right) \in$ $L_{C}^{2}[0, h] \times L_{C}^{2}[h, 1]$. Let $w_{1}$ and $w_{2}$ be nonzero constants.

We consider the pair of Sturm-Liouville equations

$$
\begin{aligned}
& L_{1} y_{1} \equiv-y_{1}^{\prime \prime}+q_{1}(x) y_{1}=\lambda y_{1}, \quad 0 \leq x \leq h, \\
& L_{2} y_{2} \equiv-y_{2}^{\prime \prime}+q_{2}(x) y_{2}=\lambda y_{2}, \quad h \leq x \leq 1
\end{aligned}
$$

together with the matching conditions at the interface $x=h$ given by

$$
y_{1}(h)=y_{2}(h), \quad w_{1} y_{1}^{\prime}(h)=w_{2} y_{2}^{\prime}(h)
$$

where $\lambda$ is a complex constant.

Definition 1: By a solution of the problem (1)-(3), we mean a pair of functions $\left\{y_{1}, y_{2}\right\}$ satisfying the following conditions: 
(i) $y_{1} \in A C^{2}[0, h]$ and satisfies Equation (1) for almost all $x \in[0, h]$,

(ii) $y_{2} \in A C^{2}[h, 1]$ and satisfies Equation (2) for almost all $x \in[h, 1]$,

(iii) $y_{1}, y_{2}$ satisfy the matching conditions (3).

Definition 2: We say that the nontrivial pairs $\left\{y_{11}, y_{12}\right\},\left\{y_{21}, y_{22}\right\}$ where $y_{11}, y_{21}$ are defined on $[0, h]$ and $y_{12}, y_{22}$ are defined on $[h, 1]$ are linearly independent if for any two scalars $\alpha$ and $\beta$, the equations

$$
\begin{gathered}
\alpha y_{11}(x)+\beta y_{21}(x)=0 \text { for all } x \in[0, h] \text { and } \\
\alpha y_{12}(x)+\beta y_{22}(x)=0 \text { for all } x \in[h, 1]
\end{gathered}
$$

imply $\alpha=\beta=0$.

Definition 3: By a fundamental system (FS) for the problem (1)-(3), we mean a set of two linearly independent solutions of (1)-(3), which span the solution space of (1)-(3).

In Part I, we construct a FS for the problem (1)-(3) and establish certain estimates for the components of FS. In Part II, we present results concerning the location of the spectra of various associated BVPs.

\section{A Fundamental System for (1)-(3): Construction and Estimates}

For the sake of simplicity, we denote $C_{\lambda}(x)=(\cos \sqrt{\lambda} x)$ and $S_{\lambda}(x)=\frac{\sin \sqrt{\lambda} x}{\sqrt{\lambda}}$. Before
proving the main theorem, we state the following easily verified lemmas. proving the main theorem, we state the following easily verified lem
Lemma 1: Let $\left(g_{1}, g_{2}\right) \in L_{C}^{2}[0, h] \times L_{C}^{2}[h, 1]$. Then for $x \in[h, 1]$,

$$
\int_{0}^{h}\left|g_{1}(t)\right| d t+\int_{h}^{x}\left|g_{2}(t)\right| d t \leq\left(\left\|g_{1}\right\|^{2}+\left\|g_{2}\right\|^{2}\right)^{1 / 2} \sqrt{x} \text {. }
$$

Lemma 2: (A) The problem (1)-(3) along the initial conditions

$$
y_{1}(0)=1, \quad y_{1}^{\prime}(0)=0
$$

is equivalent to the Liouville integral equation

$$
\begin{aligned}
y_{1}(x)= & C_{\lambda}(x)+\int_{0}^{x} S_{\lambda}(x-t) q_{1}(t) y_{1}(t) d t, \quad 0 \leq x \leq h \\
y_{2}(x) & =y_{1}(h) C_{\lambda}(x-h)+\left(w_{1} / w_{2}\right) y_{1}^{\prime}(h) S_{\lambda}(x-h) \\
& +\int_{h}^{x} S_{\lambda}(x-t) q_{2}(t) y_{2}(t) d t, \quad h \leq x \leq 1
\end{aligned}
$$

(B) The problem (1)-(3) along with the initial conditions

$$
y_{1}(0)=0, \quad y_{1}^{\prime}(0)=1
$$

is equivalent to the Liouville integral equation 


$$
\begin{gathered}
y_{1}(x)=S_{\lambda}(x)+\int_{0}^{x} S_{\lambda}(x-t) q_{1}(t) y_{1}(t) d t, \quad 0 \leq x \leq h, \\
y_{2}(x)=y_{1}(h) C_{\lambda}(x-h)+\left(w_{1} / w_{2}\right) y_{1}^{\prime}(h) S_{\lambda}(x-h) \\
+\int_{h}^{x} S_{\lambda}(x-t) q_{2}(t) y_{2}(t) d t, \quad h \leq x \leq 1 .
\end{gathered}
$$

Theorem 1: (Construction of a FS for (1)-(3)) (A) The unique solution of initial value problem (1)-(4) is given by the pair $\left(y_{11}, y_{12}\right)$ where

$$
\begin{gathered}
y_{11}(x)=C_{\lambda}(x)+\sum_{n=1}^{\infty} \int_{0 \leq t_{1} \leq \ldots \leq t_{n} \leq t_{n+1}=x} C_{\lambda}\left(t_{1}\right) \prod_{i=1}^{n} \\
\times S_{\lambda}\left(t_{i+1}-t_{i}\right) q_{1}\left(t_{i}\right) d t_{1} \ldots d t_{n}, \quad 0 \leq x \leq h, \\
y_{12}(x)=C_{0}(x, \lambda)+\sum_{n=1}^{\infty} \quad \int_{h \leq t_{1} \leq \ldots \leq t_{n} \leq t_{n+1}=x} C_{0}\left(t_{1}, \lambda\right) \prod_{i=1}^{n} \\
\times S_{\lambda}\left(t_{i+1}-t_{i}\right) q_{2}\left(t_{i}\right) d t_{1} \ldots d t_{n}, \quad h \leq x \leq 1,
\end{gathered}
$$

where

$$
C_{0}(x, \lambda)=y_{11}(h) C_{\lambda}(x-h)+\frac{w_{1}}{w_{2}}\left(y_{11}^{\prime}(h) S_{\lambda}(x-h)\right), \quad h \leq x \leq 1 .
$$

(B) The unique solution of initial value problem (1)-(3) and (7) is given by the pair $\left(y_{21}, y_{22}\right)$ where

$$
\begin{gathered}
y_{21}(x)=S_{\lambda}(x)+\sum_{n=1}^{\infty} \int_{0 \leq t_{1} \leq \ldots \leq t_{n} \leq t_{n+1}=x} S_{\lambda}\left(t_{1}\right) \prod_{i=1}^{n} \\
\times S_{\lambda}\left(t_{i+1}-t_{i}\right) q_{1}\left(t_{i}\right) d t_{1} \ldots d t_{n}, \quad 0 \leq x \leq h, \\
y_{22}(x)=S_{0}(x, \lambda)+\sum_{n=1}^{\infty} \quad \int_{h \leq t_{1} \leq \ldots \leq t_{n} \leq t_{n+1}=x} S_{0}\left(t_{1}, \lambda\right) \prod_{i=1}^{n} \\
\times S_{\lambda}\left(t_{i+1}-t_{i}\right) q_{2}\left(t_{i}\right) d t_{1} \ldots d t_{n}, \quad h \leq x \leq 1,
\end{gathered}
$$

where

$$
S_{0}(x, \lambda)=y_{21}(h) C_{\lambda}(x-h)+\frac{w_{1}}{w_{2}}\left(y_{21}^{\prime}(h) S_{\lambda}(x-h)\right), \quad h \leq x \leq 1
$$

Here

and

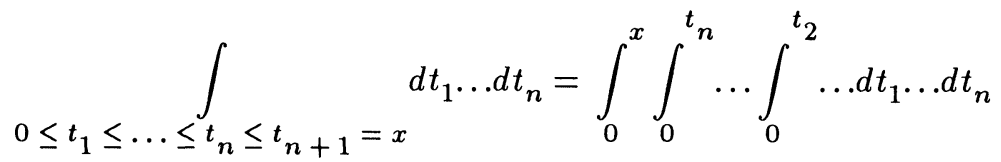




$$
\int_{h \leq t_{1} \leq \ldots \leq t_{n} \leq t_{n+1}=x} d t_{1} \ldots d t_{n}=\int_{h}^{x} \int_{h}^{t_{n}} \ldots \int_{h}^{t_{2}} \ldots d t_{1} \ldots d t_{n} .
$$

Proof: We prove part $(A)$. The proof of part $(B)$ follows similarly. Expression (10) follows from Theorem 1 (on p. 7 [7]). One can also refer to Equation (8) on page 9 [4], for the power series representations in (10) and (13). Below we derive Expression (11).

We assume that $y_{12}$ is a power series in $q_{2}$, that is

$$
y_{12}(x)=C_{0}(x, \lambda)+\sum_{n=1}^{\infty} C_{n}\left(x, \lambda, q_{2}\right), \quad h \leq x \leq 1
$$

where $C_{n}\left(x, \lambda, q_{2}\right)=\left.C_{n}\left(x, \lambda, q_{12}, \ldots, q_{n 2}\right)\right|_{q_{12}=q_{22}=\ldots=q_{n 2}=q_{2}}$, and $C_{n}\left(x, \lambda, q_{12}, \ldots\right.$, $\left.q_{n 2}\right)$, for each $x$ and $\lambda$, is a bounded, multilinear symmetric form on $L_{C}^{2}[h, 1] \times \ldots(n$ times) $\ldots \times L_{C}^{2}[h, 1]$.

Formally differentiating the power series for $y_{12}$ twice with respect to $x$ and substituting into Equation (2) then equating the terms which are homogeneous of the same degree in $q_{2}$, we obtain

$$
\begin{gathered}
-C_{0}^{\prime \prime}=\lambda C_{0} \\
-C_{n}^{\prime \prime}=\lambda C_{n}-q_{2} C_{n-1}, n \geq 1, h \leq x \leq 1
\end{gathered}
$$

In view of the matching conditions (3) to be satisfied by the pair $\left(y_{11}, y_{12}\right)$ at the interface $x=h$, we impose the following initial conditions on the $C_{n}^{\prime} \mathrm{s}$ :

$$
\begin{gathered}
C_{0}(h, \lambda)=y_{11}(h), C_{0}^{\prime}(h, \lambda)=\frac{w_{1}}{w_{2}} y_{11}^{\prime}(h) \\
C_{n}(h)=C_{n}^{\prime}(h)=0, \quad n \geq 1 .
\end{gathered}
$$

Clearly, the solution of (17) satisfying (19) is given by

$$
C_{0}(x, \lambda)=y_{11}(h) C_{\lambda}(x-h)+\frac{w_{1}}{w_{2}}\left(y_{11}^{\prime}(h) S_{\lambda}(x-h)\right) .
$$

Also the solution of (18) satisfying (20) is given by

$$
C_{n}\left(x, \lambda, q_{2}\right)=\int_{h}^{x} S_{\lambda}(x-t) q_{2}(t) C_{n-1}\left(t, \lambda, q_{2}\right) d t, \quad n \geq 1 .
$$

Proceeding by induction, we get

$$
C_{n}\left(x, \lambda, q_{2}\right)=\int_{h \leq t_{1} \leq \ldots<t_{n}<t_{n+1}=x} C_{0}\left(t_{1}, \lambda\right) \prod_{i=1}^{n} S_{\lambda}\left(t_{i+1}-t_{i}\right) q_{2}\left(t_{i}\right) d t_{1} \ldots d t_{n} .
$$

Substituting the expressions for $C_{0}, C_{n}$ in (16), we obtain (11).

Note 1: We note that

and

$$
y_{j 1}(x)=y_{j 1}\left(x, \lambda, q_{1}\right)
$$

$$
y_{j 2}(x)=y_{j 2}\left(x, \lambda, w_{1}, w_{2}, q_{1}, q_{2}\right), j=1,2 \text {. }
$$


Theorem 2: $(A)$ The formal power series for $y_{j 1}, j=1,2$ converge uniformly on bounded subsets of $[0, h] \times C \times L_{C}^{2}[0, h]$ and

$$
\left|y_{j 1}(x)\right| \leq \exp \left(|\operatorname{Im} \sqrt{\lambda}| x+\left\|q_{1}\right\| \sqrt{x}\right), \quad 0 \leq x \leq h .
$$

(B) The formal power series for $y_{j 2}, j=1,2$ converge uniformly on bounded subset of $[h, 1] \times C \times(C \backslash\{0\}) \times\left(C \backslash B_{r}(0)\right) \times L_{C}^{2}[0, h] \times L_{C}^{2}[h, 1]$, for any $r>0$ and

$$
\left|y_{j 2}(x)\right| \leq\left(1+\left|1-\frac{w_{1}}{w_{2}}\right|\right) \exp \left(|\operatorname{Im} \sqrt{\lambda}| x+\left(\left\|q_{1}\right\|^{2}+\left\|q_{2}\right\|^{2}\right)^{1 / 2} \sqrt{x}\right), \quad h \leq x \leq 1 .
$$

Proof: The proof of part $(A)$ follows from Theorem 1 (on p. 7 [7]). Below we shall derive the estimate for $\left|y_{12}(x)\right|$. The estimate for $\left|y_{22}(x)\right|$ can be derived along similar lines.

We note that

and for $0 \leq x \leq 1$,

$$
\left|C_{\lambda}(x)\right| \leq \exp (|\operatorname{Im} \sqrt{\lambda}| x)
$$

$$
\left|S_{\lambda}(x)\right| \leq \exp (|\operatorname{Im} \sqrt{\lambda}| x), \quad(\text { see p. } 8[7]) .
$$

Substituting the series (10) and its derived series for $y_{11}(h)$ and $y_{11}^{\prime}(h)$, respectively, into Expression (12), regrouping the terms, taking modulus and using the triangle inequality, we obtain

$$
\begin{aligned}
& \left|C_{0}(x, \lambda)\right| \leq\left|C_{\lambda}(x)+\left(1-\frac{w_{1}}{w_{2}}\right) \operatorname{Sin}(\sqrt{\lambda}(x-h)) \operatorname{Sin}(\sqrt{\lambda} h)\right|+\sum_{n=1}^{\infty} \\
& \int_{0 \leq t_{1}<\ldots<t_{n}<t_{n+1}=h} C_{\lambda}\left(t_{1}\right) q_{1}\left(t_{n}\right) \prod_{i=1}^{n-1} S_{\lambda}\left(t_{i+1}-t_{i}\right) q_{1}\left(t_{i}\right) \\
& \times\left[S_{\lambda}\left(x-t_{n}\right)+\left(\frac{w_{1}}{w_{2}}-1\right) S_{\lambda}(x-h) C_{\lambda}\left(h-t_{n}\right)\right] d t_{1} \ldots d t_{n} \\
& \leq\left(1+\left|1-\frac{w_{1}}{w_{2}}\right|\right) \exp (|\operatorname{Im} \sqrt{\lambda}| x) \\
& \times\left[1+\sum_{n=1}^{\infty} \int_{0 \leq t_{1} \leq \ldots<t_{n} \leq t_{n+1}=h} \prod_{i=1}^{n}\left|q_{1}\left(t_{i}\right)\right| d t_{1} \ldots d t_{n}\right] \\
& =\left(1+\left|1-\frac{w_{1}}{w_{2}}\right|\right) \exp (|\operatorname{Im} \sqrt{\lambda}| x) \times\left[1+\sum_{n=1}^{\infty} \frac{1}{n !}\left(\int_{0}^{h}\left|q_{1}(t)\right| d t\right)^{n}\right](\text { see p. } 8[7]) \\
& \left|C_{0}(x, \lambda)\right| \leq\left(1+\left|1-\frac{w_{1}}{w_{2}}\right|\right) \exp \left[|\operatorname{Im} \sqrt{\lambda}| x+\int_{0}^{h}\left|q_{1}(t)\right| d t\right], h \leq x \leq 1 .
\end{aligned}
$$

Finally from Equation (11), we get 


$$
\begin{aligned}
& \left|y_{12}(x)\right| \leq\left|C_{0}(x, \lambda)\right|+\sum_{n=1}^{\infty} \\
& \times\left|C_{0}\left(t_{1}, \lambda\right)\right| \prod_{i=1}^{n} S_{\lambda}\left(t_{i-1}-t_{i}\right)\left|q_{2}\left(t_{i}\right)\right| d t_{1} \ldots d t_{n} \\
& \leq\left(1+\left|1-\frac{w_{1}}{w_{2}}\right|\right) \exp \left[|\operatorname{Im} \sqrt{\lambda}| x+\int_{0}^{h}\left|q_{1}(t)\right| d t\right] \\
& \times\left[1+\sum_{n=i}^{\infty}\right. \\
& \left.\int_{h \leq t_{1} \leq \ldots \leq t_{n+1}=x^{i=1}} \prod_{n}^{n}\left|q_{2}\left(t_{i}\right)\right| d t_{1} \ldots d t_{n}\right] \\
& \text { (using (21) and simplifying) } \\
& \begin{aligned}
= & \left(1+\left|1-\frac{w_{1}}{w_{2}}\right|\right) \exp \left[|\operatorname{Im} \sqrt{\lambda}| x+\int_{0}^{h}\left|q_{1}(t)\right| d t+\int_{h}^{x}\left|q_{2}(t)\right| d t\right] \text { (as before) } \\
& \leq\left(1+\left|1-\frac{w_{1}}{w_{2}}\right|\right) \exp \left[|\operatorname{Im} \sqrt{\lambda}| x+\left(\left\|q_{1}\right\|^{2}+\left\|q_{2}\right\|^{2}\right)^{1 / 2} \sqrt{x}\right], h \leq x \leq 1
\end{aligned}
\end{aligned}
$$

(by Lemma 1).

The above estimate readily implies the uniform convergence of the power series for $y_{12}$.

Note 2: Theorem 2 and Lemma 2 readily imply the uniqueness of the solution stat in Theorem 1. Moreover, every solution $\left(y_{1}, y_{2}\right)$ of the problem (1)-(3) is uniquely expressed in the form

$$
\begin{array}{ll}
y_{1}(x)=y_{1}(0) y_{11}(x)+y_{1}^{\prime}(0) y_{21}(x), & 0 \leq x \leq h \\
y_{2}(x)=y_{1}(0) y_{12}(x)+y_{1}^{\prime}(0) y_{22}(x), & h \leq x \leq 1
\end{array}
$$

Lastly, we prove the following theorem on the asymptotic estimates for the components of the FS.

Theorem 3: $(A)$ On $[0, h] \times C \times L_{C}^{2}[0, h]$,

(ii) $\quad\left|y_{21}(x)-\frac{\sin \sqrt{\lambda} x}{\sqrt{\lambda}}\right| \leq \frac{1}{|\lambda|} \exp \left(|\operatorname{Im} \sqrt{\lambda}| x+\left\|q_{1}\right\| \sqrt{x}\right)$,

(iii) $\left|y_{11}^{\prime}(x)+\sqrt{\lambda} \sin \sqrt{\lambda} x\right| \leq\left\|q_{1}\right\| \exp \left(|\operatorname{Im} \sqrt{\lambda}| x+\left\|q_{1}\right\| \sqrt{x}\right)$,

(iv) $\quad\left|y_{21}^{\prime}(x)-\cos \sqrt{\lambda} x\right| \leq \frac{\left\|q_{1}\right\|}{|\sqrt{\lambda}|} \exp \left(|\operatorname{Im} \sqrt{\lambda}| x+\left\|q_{1}\right\| \sqrt{x}\right)$.

(B) On $[h, 1] \times C \times(C \backslash\{0\}) \times\left(C \backslash B_{r}(0)\right) \times L_{C}^{2}[0, h] \times L_{C}^{2}[h, 1]$,

(v) $\quad\left|y_{12}(x)-\cos \lambda x\right| \leq\left|1-\frac{w_{1}}{w_{2}}\right| \exp (|\operatorname{Im} \sqrt{\lambda}| x)$ 


$$
+\left(\frac{1+\left|1-\frac{w_{1}}{w_{2}}\right|}{|\sqrt{\lambda}|}\right) \exp \left(|\operatorname{Im} \sqrt{\lambda}| x+\left(\left\|q_{1}\right\|^{2}+\left\|q_{2}\right\|^{2}\right)^{1 / 2} \sqrt{x}\right)
$$

(vi) $\quad\left|y_{22}(x)-\frac{\sin \sqrt{x}}{\sqrt{\lambda}}\right| \leq \frac{1}{|\sqrt{\lambda}|}\left|1-\frac{w_{1}}{w_{2}}\right| \exp (|\operatorname{Im} \sqrt{\lambda}| x)$

$$
+\left(\frac{1+\left|1-\frac{w_{1}}{w_{2}}\right|}{|\lambda|}\right) \exp \left(|\operatorname{Im} \sqrt{\lambda}| x+\left(\left\|q_{1}\right\|^{2}+\left\|q_{2}\right\|^{2}\right)^{1 / 2} \sqrt{x}\right)
$$

(vii) $\quad\left|y_{12}^{\prime}(x)+\sqrt{\lambda} \sin \sqrt{x}\right| \leq|\sqrt{\lambda}|\left|1-\frac{w_{1}}{w_{2}}\right| \exp (|\operatorname{Im} \sqrt{\lambda}| x)$

$+\left(1+\left|1-\frac{w_{1}}{w_{2}}\right|\right)\left(\left\|q_{1}\right\|^{2}+\left\|q_{2}\right\|^{2}\right)^{1 / 2} \exp \left(|\operatorname{Im} \sqrt{\lambda}| x+\left(\left\|q_{1}\right\|^{2}+\left\|q_{2}\right\|^{2}\right)^{1 / 2} \sqrt{x}\right)$

(viii) $\left|y_{22}^{\prime}(x)-\cos \sqrt{\lambda} x\right| \leq\left|1-\frac{w_{1}}{w_{2}}\right| \exp (|\operatorname{Im} \sqrt{\lambda}| x)$

$$
\begin{aligned}
& +\frac{1}{|\sqrt{\lambda}|}\left(1+\left|1-\frac{w_{1}}{w_{2}}\right|\right)\left(\left\|q_{1}\right\|^{2}+\left\|q_{2}\right\|^{2}\right)^{1 / 2} \\
& \times \exp \left(|\operatorname{Im} \sqrt{\lambda}| x+\left(\left\|q_{1}\right\|^{2}+\left\|q_{2}\right\|^{2}\right)^{1 / 2} \sqrt{x}\right) .
\end{aligned}
$$

Proof: The proof of part $(A)$ follows from Theorem 3 (p. 13 [7]). We establish inequalities $(v)$ and $(v i i)$ of $(B)$. Inequalities $(v i)$ and $(v i i i)$ can be established similarly.

(v) From

$$
\begin{gathered}
+\sum_{n=1}^{\infty} \begin{array}{c}
C_{0}(x, \lambda)=C_{\lambda}(x)+\left(1-\frac{w_{1}}{w_{2}}\right) \operatorname{Sin}(\sqrt{\lambda}(x-h)) \operatorname{Sin}(\sqrt{\lambda} h) \\
0 \leq t_{1} \leq \ldots \leq t_{n} \leq t_{n+1}=h \\
\end{array} \int_{\lambda}\left(t_{1}\right) q_{1}\left(t_{n}\right) \prod_{i=1}^{n-1} s_{\lambda}\left(t_{i+1}-t_{i}\right) q_{1}\left(t_{i}\right) \\
\times\left[S_{\lambda}\left(x-t_{n}\right)+\left(\frac{w_{1}}{w_{2}}-1\right) S_{\lambda}(x-h) C_{\lambda}\left(h-t_{n}\right)\right] d t_{1} \ldots d t_{n}
\end{gathered}
$$

it follows by using the same type of estimating as in (21) that

$$
\begin{gathered}
\left|C_{0}(x, \lambda)-\cos \sqrt{\lambda} x\right| \\
\leq\left|1-\frac{w_{1}}{w_{2}}\right| \exp (|\operatorname{Im} \sqrt{\lambda}| x)+\frac{1}{\mid \sqrt{\lambda}}\left(1+\left|1-\frac{w_{1}}{w_{2}}\right|\right) \exp \left(|\operatorname{Im} \sqrt{\lambda}|+\int_{0}^{h}\left|q_{1}(t)\right| d t\right) .
\end{gathered}
$$




$$
\mid y_{12}(x)-\cos (\sqrt{\lambda} x)
$$

$\leq\left|C_{0}(x, \lambda)-C_{\lambda}(x)\right|$

$$
\begin{gathered}
+\sum_{n=1}^{\infty}\left|\int_{h \leq t_{1} \ldots<t_{n+1}=x} C_{0}\left(t_{1}, \lambda\right) \prod_{i=1}^{n} S_{\lambda}\left(t_{i+1}-t_{i}\right) q_{2}\left(t_{i}\right) d t_{1} \ldots d t_{n}\right| \\
\leq\left|1-\frac{w_{1}}{w_{2}}\right| \exp (|\operatorname{Im} \sqrt{\lambda}|) \\
\frac{1}{\sqrt{\lambda}}\left(1+\left|1-\frac{w_{1}}{w_{2}}\right|\right) \exp \left(|\operatorname{Im} \sqrt{\lambda}| x+\int_{0}^{h}\left|q_{1}(t)\right| d t\right)
\end{gathered}
$$

(by using (21), (23) and the second sum estimation in (22))

$$
\leq\left|1-\frac{w_{1}}{w_{2}}\right| \exp (|\operatorname{Im} \sqrt{\lambda}| x)+\frac{1}{|\sqrt{\lambda}|}\left(1+\left|1-\frac{w_{1}}{w_{2}}\right|\right) \exp \left(|\operatorname{Im} \sqrt{\lambda}| x+\left(\left\|q_{1}\right\|^{2}+\left\|q_{2}\right\|^{2}\right)^{1 / 2} \sqrt{x}\right)
$$

(by Lemma 1).

(vii) Differentiating integral equation (6) for $y_{12}$ with respect to $x$, inserting $y_{11}(h), y_{11}^{\prime}(h)$ from integral equation (5), and simplifying we obtain

$$
\begin{gathered}
y_{12}^{\prime}(x)=-\sqrt{\lambda}\left[\cos (\sqrt{\lambda} h) \sin (\sqrt{\lambda}(x-h))+\frac{w_{1}}{w_{2}} \sin (\sqrt{\lambda} h) \cos (\sqrt{\lambda}(x-h))\right] \\
+\int_{0}^{h} q_{1}(t) y_{11}(t)\left[-\sin (\sqrt{\lambda}(h-t)) \sin (\sqrt{\lambda}(x-h))+\frac{w_{1}}{w_{2}} \cos (\sqrt{\lambda}(h-t)) \cos (\sqrt{\lambda}(x-h))\right] d t \\
+\int_{h}^{x} C_{\lambda}(x-t) q_{2}(t) y_{12}(t) d t
\end{gathered}
$$

Hence

$$
\begin{gathered}
\left|y_{12}^{\prime}(x)+\sqrt{\lambda} \sin (\sqrt{\lambda} x)\right| \\
\leq|\sqrt{\lambda}|\left|1-\frac{w_{1}}{w_{2}}\right| \exp (|\operatorname{Im} \sqrt{\lambda}| x) \\
+\int_{0}^{h} \exp \left(|\operatorname{Im} \sqrt{\lambda}| t+\left\|q_{1}\right\|\right)\left(1+\left|1-\frac{w_{1}}{w_{2}}\right| \exp (|\operatorname{Im} \sqrt{\lambda}|(x-t))\right)\left|q_{1}(t)\right| d t
\end{gathered}
$$$$
+\int_{h}^{x} \exp (|\operatorname{Im} \sqrt{\lambda}|(x-t))\left(1+\left|1-\frac{w_{1}}{w_{2}}\right|\right) \exp \left(|\operatorname{Im} \sqrt{\lambda}| t+\left(\left\|q_{1}\right\|^{2}+\left\|q_{2}\right\|^{2}\right)^{1 / 2} \sqrt{t}\right)
$$$$
\times\left|q_{2}(t)\right| d t \text { (by Theorem 2) }
$$$$
\leq|\sqrt{\lambda}|\left|1-\frac{w_{1}}{w_{2}}\right| \exp (|\operatorname{Im} \sqrt{x}| x)
$$ 


$$
+\left(1+\left|1-\frac{w_{1}}{w_{2}}\right|\right)\left(\left\|q_{1}\right\|^{2}+\left\|q_{2}\right\|^{2}\right)^{1 / 2} \exp \left(|\operatorname{Im} \sqrt{\lambda}| x+\left(\left\|q_{1}\right\|^{2}+\left\|q_{2}\right\|^{2}\right)^{1 / 2} \sqrt{x}\right)
$$

(by Lemma 1).

Note 3: It follows from standard results for initial value problems that $y_{i j}$, $i, j=1,2$ and their derivatives are analytic functions of $\lambda$.

\section{Acknowledgement}

The authors are extremely grateful to the referee for the useful suggestions and comments which resulted in the present form of the paper. The authors dedicate the work to the Chancellor of the Institute Bhagawan Sri Satya Sai Baba.

\section{References}

[1] Boyles, C.A., Acoustic Waveguides, Applications to Oceanic Sciences, Wiley, New York 1984.

[2] Ghosh, P.K., The Mathematics of Waves and Vibrations, MacMillan, India 1975.

[3] Bhaskar, T.G., A Study of Mixed Linear Regular Ordinary Differential Operators, Ph.D. Thesis, Sri Sathya Sai Institute of Higher Learning 1990.

[4] Borg, G., Eine Umkehrung der Sturm-Liouvillschen Eigenwertaufgabe: Bestimmung der Differentialgleichung durch die Eigenwerte, Acta. Math. 78 (1946), 196.

[5] Hald, O.H., Discontinuous Inverse eigenvalue problems, Comm. Pure and Appl. Math XXXVII (1984), 339-377.

[6] Harris, B.J., A series solution for certain Riccatti equations with applications to Sturm-Liouville problems, J. Math. Anal. Appl. 137 (1989), 462-470.

[7] Poschel, J. and Trubowitz, E., Inverse Spectral Theory, Academic Press, New York 1987.

[8] Venkatesulu, M. and Bhaskar, T.G., Self-adjoint boundary value problems associated with a pair of mixed linear ordinary differential equations, J. Math. Anal. Appl. 144:2 (1989), 322-341.

[9] Wang, C.-T., Applied Elasticity, McGraw-Hill, New York 1953. 


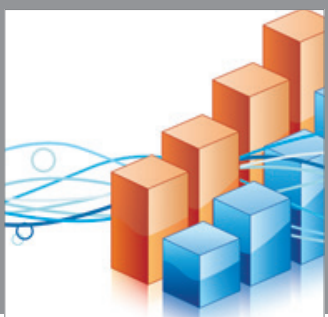

Advances in

Operations Research

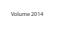

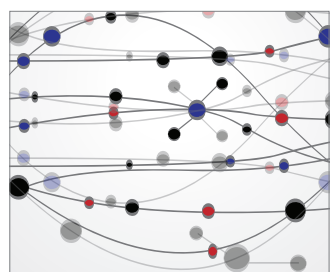

\section{The Scientific} World Journal
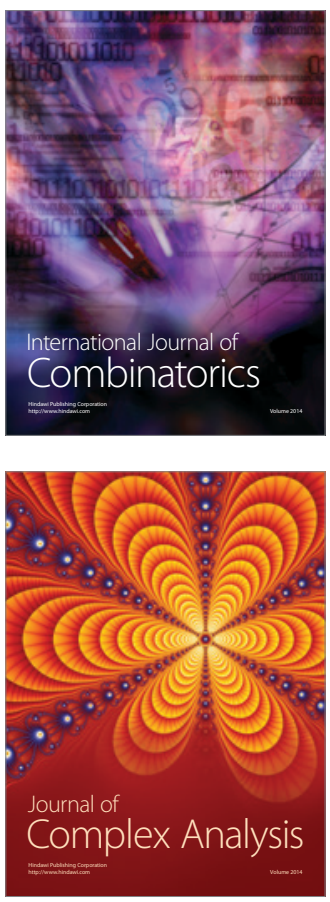

International Journal of

Mathematics and

Mathematical

Sciences
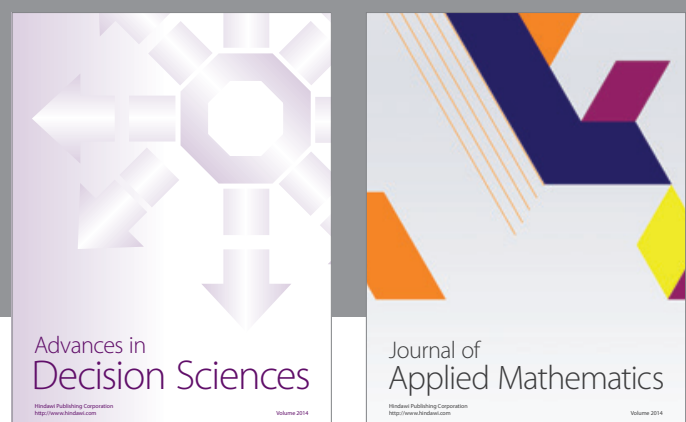

Journal of

Applied Mathematics
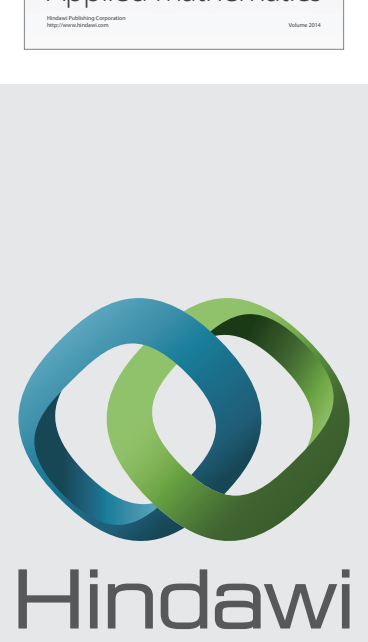

Submit your manuscripts at http://www.hindawi.com
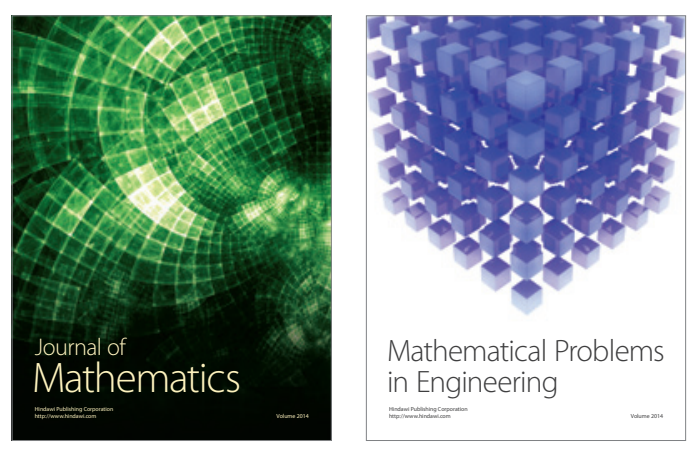

Mathematical Problems in Engineering
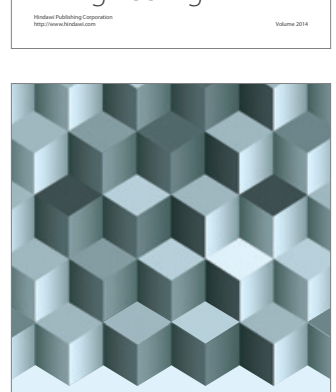

Journal of

Function Spaces
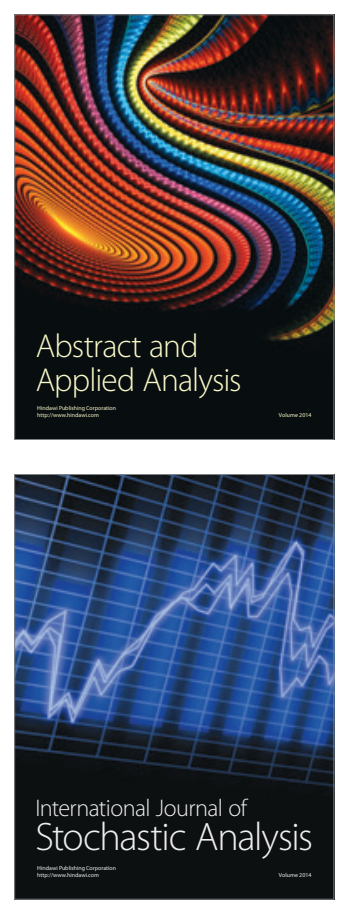

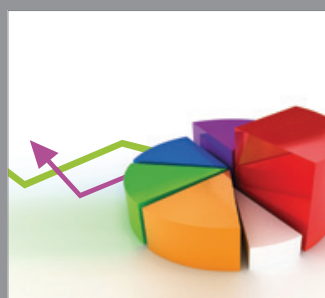

ournal of

Probability and Statistics

Promensencen
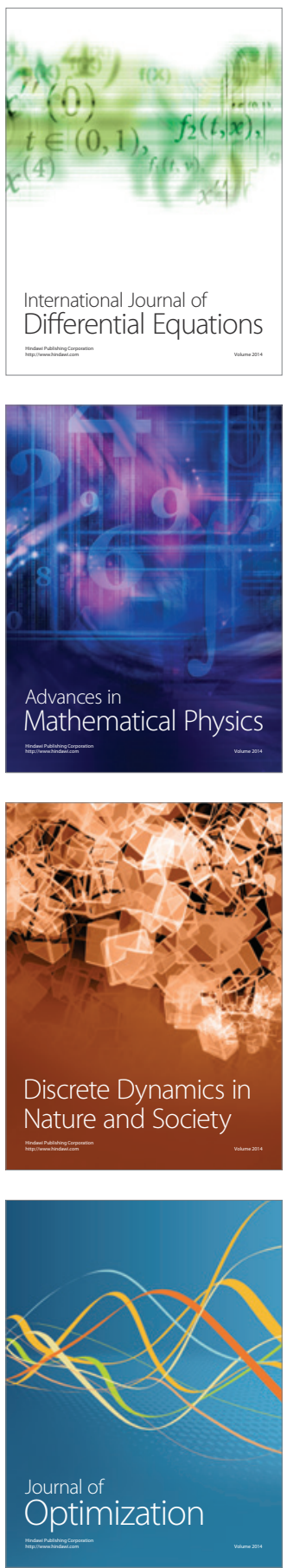